\title{
ON VECTOR STATES AND SEPARABLE $C^{*}$-ALGEBRAS
}

\author{
JOEL ANDERSON
}

\begin{abstract}
It is proved that the set of states on a separable $C^{*}$-subalgebra of the Calkin algebra may be simultaneously extended to a set of equivalent, orthogonal, pure states on the Calkin algebra.
\end{abstract}

Let $\mathfrak{A}$ denote a separable $C^{*}$-algebra of operators acting on a separable Hilbert space $\mathcal{H}$ and suppose that $\mathfrak{A}$ contains the identity. In [2] Glimm proved that the weak*-closure of the set of vector states on $\mathfrak{A}$ (i.e., states on $\mathfrak{A}$ of the form $\omega_{x}(A)=(A x, x)$, where $A \in \mathfrak{A}$ and $x$ is a unit vector in $\left.\mathscr{H}\right)$ contains the set $\mathcal{S}(\mathfrak{A})$ of all states on $\mathfrak{A}$ which annihilate $\mathfrak{A} \cap \mathcal{K}(\mathcal{H})$. $(\mathcal{K}(\mathcal{H})$ denotes the compact operators acting on $\mathcal{H}$.) Voiculescu used this result in [3] in the proof of his noncommutative Weyl-von Neumann theorem. In this note Voiculescu's theorem shall be used to obtain a stronger version of Glimm's result: There is a sequence $\left\{\omega_{n}\right\}$ of vector states, induced by an orthonormal set of vectors in $\mathcal{H}$, such that $\mathcal{S}(\mathfrak{U})$ is contained in the weak ${ }^{*}$-closure of $\left\{\omega_{n}\right\}$. (It should be noted that Glimm's theorem holds without any separability assumptions so that the theorem to be proved here is stronger only in the separable case.)

This theorem, together with a theorem from [1], yields a somewhat surprising corollary: There is a set $\mathcal{E}$ consisting of equivalent, orthogonal, pure states on $\mathscr{B}(\mathcal{H})$, the bounded linear operators on $\mathcal{H}$, such that every state in $\mathcal{S}(\mathfrak{A})$ is a restriction of a state in $\mathcal{E}$. In particular, if $f$ is any state on a separable $C^{*}$-subalgebra of the Calkin algebra, $\mathscr{B}(\mathcal{H}) / \mathscr{K}(\mathcal{H})$, then there is a pure state $g$ on the Calkin algebra which extends $f$.

To prove the theorem, note that since $\mathfrak{A}$ is separable, $s(\mathfrak{A})$ is weak*-metrizable and compact and so contains a countable dense set, say $\left\{f_{n}\right\}$.

Let $p$ denote the canonical homomorphism of $\mathscr{B}(\mathcal{H})$ onto the Calkin algebra. Then each state $f_{n}$ determines a state $g_{n}$ on $p(\mathfrak{U})$ such that $f_{n}=g_{n} \circ p$. Let $\left\{\pi_{n}, \mathcal{H}_{n}, x_{n}\right\}$ denote the G.N.S. representation of $p(\mathfrak{A})$ constructed from $g_{n}$. Then $\pi_{n}$ is a ${ }^{*}$-homomorphism of $p(\mathfrak{U})$ into $\mathscr{B}\left(\mathcal{H}_{n}\right)$ and $f_{n}(A)=g_{n} \circ p(A)$ $=\left(\pi_{n} \circ p(A) x_{n}, x_{n}\right)$ for each $A$ in $\mathfrak{A}$. Let $\pi$ denote the representation of $p(\mathfrak{A})$ obtained by taking the direct sum of the $\pi_{n}$ 's, so that $\pi$ maps $p(\mathfrak{A})$ into $\mathscr{B}\left(\Sigma \oplus \mathcal{K}_{n}\right)$. By Voiculescu's theorem, there is a unitary transformation $U$ of

Received by the editors September 30, 1976.

AMS (MOS) subject classifications (1970). Primary $46 \mathrm{~L} 05$.

Key words and phrases. $C^{*}$-algebra, state, pure state, vector state, Calkin algebra.

(1) American Mathematical Society 1977 
$\mathcal{H}$ onto $\mathcal{H} \oplus \Sigma \oplus \mathcal{K}_{n}$ such that $A-U^{*}(A \oplus \pi \circ p(A)) U \in \mathcal{K}(\mathcal{H})$ for all $A$ in $\mathfrak{A}$. Write $e_{n}=U^{*} x_{n}$ for $n=1,2, \ldots$ Then $\left\{e_{n}\right\}$ is an orthonormal sequence in $\mathcal{H}$ and the vector states $\omega_{n}=\omega_{e_{n}}, n=1,2, \ldots$, have the desired property. Indeed, if $f \in \mathcal{S}(\mathfrak{R})$, choose an infinite subsequence $\left\{f_{n}\right\}$ of $\left\{f_{n}\right\}$ which converges to $f$ in the weak*-topology. Fix $A$ in $\mathscr{U}$. Then

$$
\begin{aligned}
f(A) & =\lim _{j} f_{n^{\prime}}(A)=\lim _{j} g_{n_{j}} \circ p(A)=\lim _{j}\left(\pi_{n_{j}} \circ p(A) x_{n_{j}}, x_{n_{j}}\right) \\
& =\lim _{j}\left(U^{*}(A \oplus \pi \circ p(A)) U e_{n_{j}}, e_{n_{j}}\right)=\lim _{j} \omega_{n_{j}}(A)+\lim _{j}\left(K e_{n}, e_{n_{j}}\right),
\end{aligned}
$$

where $K$ is the compact operator $U^{*}(A \oplus \pi \circ p(A)) U-A$. Since $\left\{e_{n}\right\}$ converges weakly to zero and $K$ is compact, $\left\|K e_{n}\right\| \rightarrow 0$ as $j \rightarrow \infty$. Hence, $f(A)=\lim _{j} \omega_{n}(A)$ for all $A$ in $\mathfrak{A}$, as desired.

To prove the corollary, choose a sequence $\left\{\omega_{n}\right\}$ of vector states induced by an orthonormal sequence $\left\{e_{n}\right\}$ such that each $f$ in $\mathcal{S}(\mathfrak{U})$ is the weak*-limit of a subsequence of the $\omega_{n}$ 's. Fix a free ultrafilter $\mathcal{Q}$ on the natural members $\Re$ and define a state $g$ on $\mathscr{B}(\mathcal{H C})$ by $g(T)=\lim _{2} \omega_{n}(T)$. For each permutation $\alpha$ of $N$ define a unitary operator $U_{\alpha}$ on $\mathcal{H}$ by $U_{\alpha} e_{n}=e_{\alpha(n)}, n=1,2, \ldots$, and define the state $g_{\alpha}$ on $\mathscr{B}(\mathcal{H C})$ by $g_{\alpha}(T)=g\left(U_{\alpha}^{*} T U_{\alpha}\right)=\lim _{2} \omega_{\alpha(n)}(T)$. (Adding vectors if necessary, we may assume that $\left\{e_{n}\right\}$ is a basis for $\mathcal{H C}^{\mathcal{C}}$.) Then the set $\mathcal{E}=\left\{g_{\alpha}: \alpha\right.$ is a permutation of $\left.\mathcal{T}\right\}$ has the desired properties. Indeed, by [1, Corollary 3] $g$, and hence each $g_{\alpha}$, is a pure state on $\mathscr{B}(\mathcal{H C})$ (because $\left\{e_{n}\right\}$ is an orthonormal sequence). Thus, $\mathcal{E}$ consists of equivalent pure states. Further, if $\alpha$ and $\beta$ are permutations of $\Re$ such that $g_{\alpha}$ and $g_{\beta}$ are distinct elements of $\mathcal{E}$, then there are disjoint subsets $\sigma$ and $\tau$ of $\Re$ such that $\alpha^{-1}(\sigma) \in \mathcal{Q}$ and $\beta^{-1}(\tau) \in \mathcal{Q}$. If $D$ is defined by $D e_{n}=e_{n}$ for $n \in \sigma$, $D e_{n}=-e_{n}$ for $n \in \tau$ and $D e_{n}=0$ otherwise, then $D \in \mathscr{B}(\mathcal{H C}),\|D\|=1$ and $g_{\alpha}(D)-g_{\beta}(D)=2$. Hence, $\left\|g_{\alpha}-g_{\beta}\right\|=2$ and the elements of $\mathcal{E}$ are orthogonal. Finally, if $f=\lim _{j} \omega_{n_{j}}$ is a state in $\mathcal{S}(\mathfrak{U})$, then for some permutation $\alpha$ of $\Re, \alpha^{-1}\left(\left\{n_{1}, n_{2}, \ldots\right\}\right) \in \mathcal{U}$ and

$$
g_{\alpha}(A)=\lim _{\mathcal{Q}} \omega_{\alpha(n)}(A)=\lim _{j} \omega_{n_{3}}(A)=f(A)
$$

for $A \in \mathfrak{A}$. The proof is complete.

Note that the choice of $\mathscr{E}$ in the proof above is far from unique. In fact, there are $2^{c}$ disjoint sets of states on $\mathscr{B}(\mathcal{H})$ which have the desired properties. (As usual, $c$ denotes the cardinality of the continuum.) Furthermore, by altering the proof somewhat, it is possible to choose a set $\mathcal{E}^{\prime}$ of disjoint (i.e., inequivalent) pure states on $\mathfrak{B}(\mathfrak{H})$ such that $\left.\mathcal{E}^{\prime}\right|_{\mathfrak{A}}=\delta(\mathfrak{A})$.

As an example, take $\mathfrak{A}$ to be an isometric isomorphic image of $C(0,1)$, the continuous functions on the unit interval, in $\mathscr{B}(\mathcal{H})$. Then $\mathscr{U} \cap \mathscr{K}(\mathcal{H})=\{0\}$ and $\delta(\mathfrak{U})$ is the entire set of states on $\mathfrak{A}$. Hence, every state on $\mathfrak{A}$ (including integration) extends to a pure state on $\mathscr{B}(\mathcal{H})$.

In conclusion, it seems worth noting that the fact that states in $\delta(\mathfrak{A})$ extend to pure states on $\mathscr{B}(\mathcal{H})$ may be proved without recourse to Voiculescu's theorem. Indeed, by a theorem of Wils [4], if $f \in \delta(\mathfrak{U})$, then $f=\lim _{\mathcal{Q}} \omega_{x_{n}}$ 
where $Q$ is a free ultrafilter on the natural numbers and $\left\{x_{n}\right\}$ is a sequence of unit vectors in $\mathcal{H C}$ such that $\lim _{2}\left(x_{n}, y\right)=0$ for all $y$ in $\mathcal{H}$. Straightforward arguments using the separabilility of $\mathfrak{A}$ can then be used to show that $f=\lim _{n} \omega_{e_{n}}$, where $\left\{e_{n}\right\}$ is an orthonormal sequence in $\mathbb{Q}$. The proof is completed, as before, by invoking Corollary 3 of [1].

\section{REFERENCES}

1. J. Anderson, Extreme points in sets of positive linear maps on $\mathscr{B}(\mathcal{S C})$ (to appear).

2. J. Glimm, A Stone-Weierstrass theorem for $C^{*}$-algebras, Ann. of Math. (2) 72 (1960), 216-244. MR 22 \#7005.

3. D. Voiculescu, A non-commutative Weyl-von Neumann theorem, Rev. Roumaine Math. Pures Appl. 21 (1976), 97-113.

4. I. M. Wils, Stone-Cech compactifications and representations of operator algebras, Doctoral dissertation, Catholic Univ. of Nijmegin, Nijmegin, 1968. MR 37 \#4637.

Department of Mathematics, Pennsylunania State University, University Park, PennSYLVANIA 16802 